\title{
Facile synthesis of quinoxaline derivatives using o-iodoxybenzoic acid (IBX) at room temperature
}

\author{
Majid M. Heravi*, Khadijeh Bakhtiari, Maryam H. Tehrani, Negar M. Javadi, and \\ Hossien A. Oskooie
}

Department of Chemistry, School of Sciences, Azzahra University, Vanak, Tehran, Iran

E-mail: mmh1331@yahoo.com

\begin{abstract}
o-Iodoxybenzoic acid (IBX), a readily available hypervalent iodine (V) reagent, was found to be highly effective in synthesis of quinoxaline derivatives, from 1,2-diketones and $O$ phenylenediamines at room temperature in very high yields.
\end{abstract}

Keywords: Quinoxalines, 1,2-diketones, o-phenylenediamines; IBX, hypervalent iodine reagents

\section{Introduction}

The importance of hypervalent iodine reagents in organic chemistry has been demonstrated in recent years, and they have been found to have several desirable properties: they are mild, selective, efficient and eco-friendly. ${ }^{1,2}$ 2-Iodoxybenzoic acid (IBX) has been developed as a powerful reagent for several organic transformations, and a recent surge in interest was driven by the publication of an improved method for its synthesis. ${ }^{3}$ IBX is a powerful single-electron transfer oxidant that readily accepts a new heteroatom-based ligand, and has been applied successfully for the construction of novel heterocycles. ${ }^{4}$

IBX oxidizes primary and secondary alcohols to the corresponding aldehydes and ketones, without any over-oxidation, in DMSO at room temperature. ${ }^{5,6}$ IBX has been used in the oxidation of oximes and tosyl hydrazones to the corresponding carbonyl compounds, ${ }^{7}$ and the oxidation of saturated alcohols and carbonyl compounds. ${ }^{8}$ It also reacted with certain unsaturated N-aryl amides to form novel heterocycles such as $\delta$-lactams, cyclic urethanes, hydroxylamine and amino sugar building blocks. ${ }^{9,10}$ Other applications of IBX include the oxidation of carbonyl compounds $^{11}$ and their silyl enol ethers ${ }^{12}$ to the corresponding $\alpha, \beta$-unsaturated compounds, regioselective oxidation of phenols to $o$-quinones ${ }^{13}$ and conversion of nitrogen- and sulfurcontaining substrates to synthetically useful intermediates. ${ }^{14}$ 
Quinoxaline derivatives are a very important class of nitrogen-containing compounds and have been widely used in dyes,${ }^{15}$ pharmaceuticals, ${ }^{16,17}$ and electrical/photochemical materials. ${ }^{18-}$ ${ }^{23}$ Quinoxaline ring moiety constitute part of the chemical structures of various antibiotics such as Echinomycin, Levomycin and Actinoleutin ${ }^{24,25}$ that are known to inhibit growth of gram positive bacteria and are active against various transplantable tumors.

A number of synthetic strategies have been developed for the preparation of substituted quinoxalines. ${ }^{26-28}$ By far, the most common method relies on the condensation of an aryl 1,2diamine with a 1,2-dicarbonyl compound in refluxing ethanol or acetic acid for 2-12h giving 34$85 \%$ yields. $^{29}$

2,3-Disubstituted quinoxalines have also been prepared by Suzuki-Miyaura coupling reaction, ${ }^{30}$ condensation of o-phenylenediamines and 1,2-dicarbonyl compounds in $\mathrm{MeOH} / \mathrm{AcOH}$ under microwave irradiation ${ }^{31}$, and iodine catalyzed cyclocondensation of 1,2dicarbonyl compounds and substituted o-phenylene diamines in $\mathrm{DMSO}^{32}$ and $\mathrm{CH}_{3} \mathrm{CN}^{33}$ As a part of our ongoing interest in synthesis of heterocyclic compounds containing nitrogen, ${ }^{34}$ we disclose herein our results for the synthesis of quinoxalines using catalytic amounts of IBX in acetic acid at room temperature (Scheme 1).

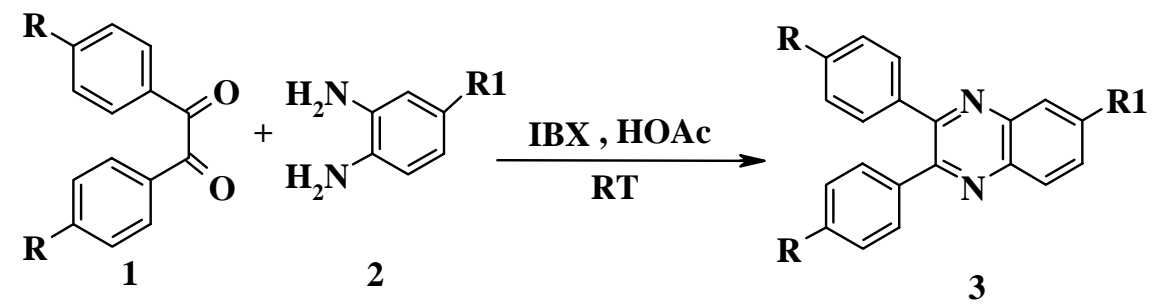

\section{Scheme 1}

\section{Results and Discussion}

Very recently we have developed a convenient and efficient procedure for synthesis of quinoxaline derivatives using cupric sulfate pentahydrate as catalyst. ${ }^{35}$

In this article we report the synthesis of quinoxaline derivatives in the presence of IBX. Compounds $3 \mathrm{a}-3 \mathrm{i}$ were obtained by the condensation of o-phenylenediamines with 1,2dicarbonyl compounds in the presence of IBX in acetic acid. The reaction proceeds very cleanly at room temperature in a one-pot procedure and free of side products. In the absence of the catalyst, the reaction did not complete even after $24 \mathrm{~h}$. Although the role of IBX is not clearly known, we suggest that it can activate the carbonyl compound and facilitate attack of the amino group to carbonyl compound. The results are shown in Table 1. The optimum yields of the products are obtained when $1 \mathrm{~mol} \%$ of IBX is used. Among solvents used such as $\mathrm{CHCl}_{3}$, $\mathrm{CH}_{3} \mathrm{CN}, \mathrm{H}_{2} \mathrm{O}$ and $\mathrm{AcOH}$ for this reaction, the later was found to be the most efficient in terms of 
reaction times and product yields. This choice of solvent can also be justified by our suggested mechanism.

Table1. Synthesis of quinoxaline derivatives catalyzed by IBX

\begin{tabular}{|c|c|c|c|c|c|c|}
\hline Entry & $\mathrm{R}$ & $\mathrm{R}^{1}$ & Product $^{\mathrm{a}}$ & Time(min) & Yield $(\%)^{\mathrm{b}}$ & m.p. $\left({ }^{\circ} \mathrm{C}\right)$ \\
\hline $3 a$ & $\mathrm{H}$ & $\mathrm{H}$ & & 5 & 98 & $128-129$ \\
\hline $3 b$ & $\mathrm{H}$ & $\mathrm{CH}_{3}$ & & 15 & 98 & $117-118$ \\
\hline $3 c$ & $\mathrm{H}$ & $\mathrm{NO}_{2}$ & & 5 & 98 & 193-194 \\
\hline $3 d$ & $\mathrm{OCH}_{3}$ & $\mathrm{H}$ & & 15 & 99 & $151-152.5$ \\
\hline $3 e$ & $\mathrm{OCH}_{3}$ & $\mathrm{CH}_{3}$ & & 10 & 98 & $125-127$ \\
\hline $3 f$ & $\mathrm{OCH}_{3}$ & $\mathrm{NO}_{2}$ & & 15 & 98 & $192-194$ \\
\hline $3 g$ & $\mathrm{~F}$ & $\mathrm{H}$ & & 5 & 99 & $135-137$ \\
\hline $3 \mathrm{~h}$ & $\mathrm{~F}$ & $\mathrm{CH}_{3}$ & & 5 & 98 & $165-167$ \\
\hline $3 \mathrm{i}$ & $\mathrm{F}$ & $\mathrm{NO}_{2}$ & & 10 & 98 & $174-176$ \\
\hline
\end{tabular}

(a) All products were well characterized using ${ }^{1} \mathrm{H}$ and IR spectra.

(b) Yields refer to isolated ure products.

Although water is a desirable solvent for chemical reactions for reasons of cost, safety and environmental concerns, use of water in this reaction gave only moderate yields of products (30\% after 24h). o-Phenylenediamines and 1,2-dicarbonyl compounds with electron-donating or electron-withdrawing groups were used. As indicated in the Table 1 both electron rich and 
electron deficient 1,2-dicarbonyl compounds worked pretty well, mostly leading to high yields of products.
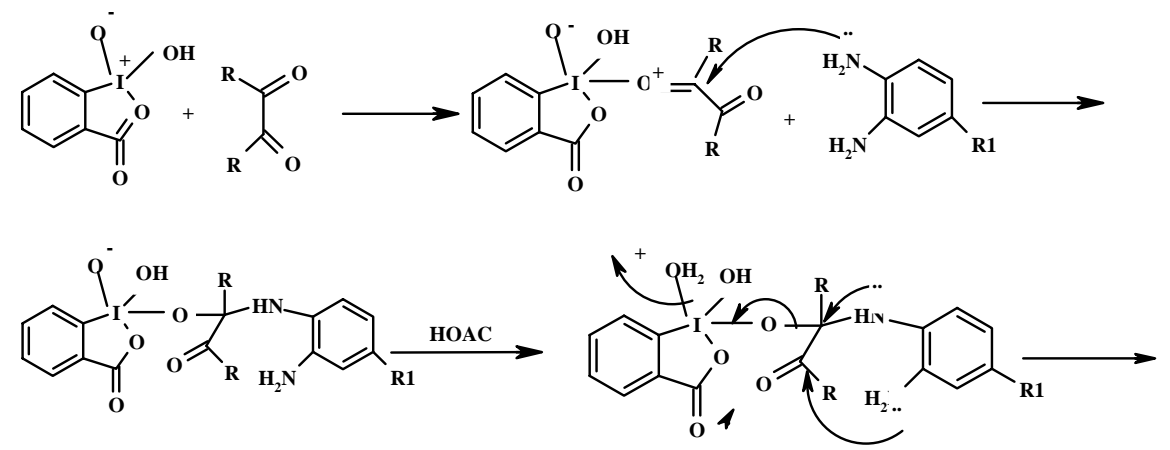<smiles>[R1]c1ccc2nc(C)c(C)nc2c1</smiles>

\section{Scheme 2}

\section{Conclusions}

In summary, we have developed the use of IBX as an inexpensive, easy to handle, non-corrosive and environmentally benign catalyst for the synthesis of quinoxalines from aromatic $o$-diamines and 1,2-dicarbonyl compounds. The advantages of the present procedure are simplicity of operation, very short reaction times compared with other procedures for the preparation of quinoxaline derivatives, and the high yields of products. In this reaction the catalyst can be recovered by filtration.

\section{Experimental Section}

General Procedures. The IBX employed for the reactions was prepared by the reported procedure. $^{27}$ (CAUTION! IBX has been reported to explode on heating.) Melting points were measured by using the capillary tube method with an electro thermal 9200 apparatus. ${ }^{1} \mathrm{H}$ NMR spectra were recorded on a Bruker AQS AVANCE-300 MHz spectrometer using TMS as an internal standard $\left(\mathrm{CDCl}_{3}\right.$ solution). IR spectra were recorded from $\mathrm{KBr}$ disk using a FT-IR Bruker Tensor 27 instrument. All products were well characterized by comparison with authentic samples by TLC, spectral and physical data. ${ }^{32,33}$ 
Preparation of quinoxalines. General procedure. A solution of aromatic $o$-diamine ( $1 \mathrm{mmol}$ ) and a 1,2-dicarbonyl compound $(1 \mathrm{mmol})$ in acetic acid $(2 \mathrm{~mL})$ was stirred at room temperature in the presence of catalytic amount of IBX $(1 \mathrm{~mol} \%, 0.028 \mathrm{~g})$. The progress of the reaction was monitored by TLC. After completion of the reaction, the catalyst was filtered off. The filtrate was successively diluted with $5 \% \mathrm{NaHCO}_{3}(5 \mathrm{~mL})$ and extracted with diethyl ether $(2 \times 5 \mathrm{~mL})$. The combined organic layers were then washed with brine $(2 \times 5 \mathrm{~mL})$, and dried over $\mathrm{MgSO}_{4}$. The solvent was evaporated under reduced pressure and the pure product was obtained without any further purification. The results are shown in Table 1.

\section{Physical and spectral data of the products}

2,3-Diphenylquinoxaline (3a). $\mathrm{Mp}=128-129^{\circ} \mathrm{C} ;{ }^{1} \mathrm{HNMR}\left(\mathrm{CDCl}_{3}, 300 \mathrm{MHz}\right) \delta(\mathrm{ppm}): 8.2(\mathrm{dd}$, $\mathrm{J}=3.43,6.30 \mathrm{~Hz}, 2 \mathrm{H}), 7.79(\mathrm{dd}, \mathrm{J}=3.43,6.30 \mathrm{~Hz}, 2 \mathrm{H}), 7.55(\mathrm{~m}, 4 \mathrm{H}), 7.39(\mathrm{~m}, 6 \mathrm{H})$; IR $(\mathrm{KBr}) v_{\max }$ $\left(\mathrm{cm}^{-1}\right): 3055,1541,1345,768,729$.

6-Methyl-2,3-diphenylquinoxaline (3b). $\mathrm{Mp}=117-118^{\circ} \mathrm{C} ;{ }^{1} \mathrm{HNMR}\left(\mathrm{CDCl}_{3}, 300 \mathrm{MHz}\right) \delta(\mathrm{ppm})$ : $8.1(\mathrm{~d}, \mathrm{~J}=8.55 \mathrm{~Hz}, 1 \mathrm{H}), 7.96(\mathrm{~s}, 1 \mathrm{H}), 7.63(\mathrm{dd}, \mathrm{J}=1.72,8.55 \mathrm{~Hz}, 1 \mathrm{H}), 7.5(\mathrm{~m}, 4 \mathrm{H}), 7.35$ (m, 6H), 2.6 (s, 3H); IR (KBr) $v_{\max }\left(\mathrm{cm}^{-1}\right): 3063,1660,1592,1210,874,719,640$.

6-Nitro-2,3-diphenylquinoxaline (3c). $\mathrm{Mp}=193-194{ }^{\circ} \mathrm{C} ;{ }^{1} \mathrm{HNMR}\left(\mathrm{CDCl}_{3}, 300 \mathrm{MHz}\right) \delta(\mathrm{ppm})$ : $9.2(\mathrm{~d}, \mathrm{~J}=2.38 \mathrm{~Hz}, 1 \mathrm{H}), 8.53$ (dd, J=2.38, 9.10Hz, 1H,), 8.39 (d, J=9.10Hz, 1H), 7.6 (m, 4H), 7.42 (m, 6H); IR (KBr) $v_{\max }\left(\mathrm{cm}^{-1}\right): 3057,2935,1621,1341,1135,699$.

2,3-Bis(4-methoxy-phenyl) quinoxaline (3d). $\mathrm{Mp}=151-152{ }^{\circ} \mathrm{C} ;{ }^{1} \mathrm{HNMR}\left(\mathrm{CDCl}_{3}, 300 \mathrm{MHz}\right)$ $\delta(\mathrm{ppm}): 8.15(\mathrm{dd}, \mathrm{J}=3.44,6.32 \mathrm{~Hz}, 2 \mathrm{H}), 7.7(\mathrm{dd}, \mathrm{J}=3.44,6.32 \mathrm{~Hz}, 2 \mathrm{H}), 7.55(\mathrm{~d}, \mathrm{~J}=8.75 \mathrm{~Hz}, 4 \mathrm{H})$, $6.89(\mathrm{~d}, \mathrm{~J}=8.73 \mathrm{~Hz}, 4 \mathrm{H}), 3.81(\mathrm{~s}, 6 \mathrm{H})$; IR $(\mathrm{KBr}) v_{\max }\left(\mathrm{cm}^{-1}\right): 3003,2931,1604,1510,1345,1057$, 876.

2,3-Bis(4-methoxy-phenyl)-6-methylquinoxaline (3e). $\mathrm{Mp}=125-127{ }^{\circ} \mathrm{C} ;{ }^{1} \mathrm{HNMR}\left(\mathrm{CDC}_{3}, 300\right.$ MHz) $\delta(\mathrm{ppm}): 8.05(\mathrm{~d}, \mathrm{~J}=8.52 \mathrm{~Hz}, 1 \mathrm{H}), 7.92$ (s, 1H), $7.58(\mathrm{dd}, \mathrm{J}=1.55,8.50 \mathrm{~Hz}, 1 \mathrm{H}), 7.48(\mathrm{~d}, \mathrm{~J}=$ $7.64 \mathrm{~Hz}, 4 \mathrm{H}), 6.9(\mathrm{~d}, \mathrm{~J}=8.75 \mathrm{~Hz}, 4 \mathrm{H}), 3.9(\mathrm{~s}, 6 \mathrm{H}), 2.6(\mathrm{~s}, 3 \mathrm{H})$; IR $(\mathrm{KBr}) v_{\max }\left(\mathrm{cm}^{-1}\right): 2925,2580$, 1657, 1597, 1264, 1159, 833, 696.

2,3-Bis(4-methoxy-phenyl)-6-nitroquinoxaline (3f). $\mathrm{Mp}=192-194{ }^{\circ} \mathrm{C} ;{ }^{1} \mathrm{HNMR}\left(\mathrm{CDC1}_{3}, 300\right.$ MHz) $\delta(\mathrm{ppm}): 9.1(\mathrm{~d}, \mathrm{~J}=2.44 \mathrm{~Hz} 1 \mathrm{H}), 8.49(\mathrm{dd}, \mathrm{J}=2.46,9.14 \mathrm{~Hz}, 1 \mathrm{H}), 8.24$ (d, J=9.15Hz, 1H), $7.56(\mathrm{~m}, 4 \mathrm{H}), 6.98(\mathrm{~d}, \mathrm{~J}=7.9 \mathrm{~Hz}, 4 \mathrm{H}), 3.9$ (s, 6H); IR (KBr) $v_{\max }\left(\mathrm{cm}^{-1}\right): 2924,1337,1169,1021$, 835.

2,3-Bis(4-flouro-phenyl)quinoxaline (3g). $\mathrm{Mp}=135-137{ }^{\circ} \mathrm{C} ;{ }^{1} \mathrm{HNMR}\left(\mathrm{CDCl}_{3}, 300 \mathrm{MHz}\right)$ $\delta(\mathrm{ppm}): 8.2(\mathrm{dd}, \mathrm{J}=3.42,7.23 \mathrm{~Hz}, 2 \mathrm{H}), 7.82(\mathrm{dd}, \mathrm{J}=3.40,6.41 \mathrm{~Hz}, 2 \mathrm{H}), 7.52(\mathrm{dd}, \mathrm{J}=5.42,8.64 \mathrm{~Hz}$, $4 \mathrm{H}), 7.05$ (dd, J=8.63, 8.64Hz, 4H); IR (KBr) $v_{\max }\left(\mathrm{cm}^{-1}\right): 3061,1599,1555,1511,1344,1225$, $839,786$.

2,3-Bis(4-flouro-phenyl)-6-methylquinoxaline (3h). $\mathrm{Mp}=165-167{ }^{\circ} \mathrm{C} ;{ }^{1} \mathrm{HNMR}\left(\mathrm{CDCl}_{3}, 300\right.$ $\mathrm{MHz}) \delta(\mathrm{ppm}): 8.05(\mathrm{~d}, \mathrm{~J}=8.55 \mathrm{~Hz}, 1 \mathrm{H}), 7.95(\mathrm{~s}, 1 \mathrm{H}), 7.64(\mathrm{dd}, \mathrm{J}=1.5,8.53 \mathrm{~Hz}, 1 \mathrm{H}), 7.51$ (dd, $\mathrm{J}=5.56,8.26 \mathrm{~Hz}, 4 \mathrm{H}), 7.05(\mathrm{dd}, \mathrm{J}=8.63,8.63 \mathrm{~Hz}, 4 \mathrm{H}), 2.7(\mathrm{~s}, 3 \mathrm{H})$; IR $(\mathrm{KBr}) v_{\max }\left(\mathrm{cm}^{-1}\right): 3056$, $1566,1525,1384,1220,1161,837$. 
2,3-Bis (4-flouro-phenyl) 6-nitroquinoxaline (3i). $\mathrm{Mp}=174-176{ }^{\circ} \mathrm{C}$; ${ }^{1} \mathrm{HNMR}\left(\mathrm{CDC}_{3}, 300\right.$ $\mathrm{MHz}) \delta(\mathrm{ppm}): 9.1(\mathrm{~d}, \mathrm{~J}=2.41 \mathrm{~Hz}, 1 \mathrm{H}), 8.56(\mathrm{dd}, \mathrm{J}=2.32,9.20 \mathrm{~Hz}, 1 \mathrm{H}), 8.3(\mathrm{~d}, \mathrm{~J}=9.17 \mathrm{~Hz}, 1 \mathrm{H}), 7.6$ $(\mathrm{m}, 4 \mathrm{H}), 7.1(\mathrm{~m}, 4 \mathrm{H})$. IR (KBr) $v_{\max }\left(\mathrm{cm}^{-1}\right): 3089,1599,1512,1343,1231,844$.

\section{References}

1. (a) Varvoglis, A. Hypervalent Iodine in Organic Synthesis; Academic Press: London, 1997.

(b) Zhdankin, V. V.; Stang, P., J. Chem. Rev., 2002, 102, 2523. (c) Ladziata, U.; Zhdankin, V. V. Arkivoc 2006, (ix), 26.

2. Heravi, M. M.; Tajbakhsh, M.; Ghassemzedeh, M. Z., Naturforschung 1999, 54, 394.

3. Frigero, M.; Santagostino, M.; Sputore, S. J. Org. Chem. 1999, 64, 4537.

4. Kumar, I. Synlett 2005, 1488.

5. Frigero, M.; Santagostino, M. Tetrahedron Lett. 1994, 35, 8019.

6. De Munari, S.; Frigero, M.; Santagostino, M. J. Org. Chem. 1996, 61, 9272.

7. Bose, D. S.; Shrinivas, P. Synlett 1998, 977.

8. Nicolaou, K. C.; Zhong, Y.-L.; Baran, P. S. J. Am. Chem. Soc. 2000, 122, 7596.

9. Nicolaou, K. C.; Zhong, Y.-L.; Baran, P. S. Angew.Chem. Int. Ed. 2000, 39, 625.

10. Nicolaou, K. C.; Baran, P. S.; Zhong, Y.-L.; Barluenga, S.; Hunt, K. W.; Karnich, R.; Vega, J. A. J. Am. Chem. Soc. 2002, 124, 2233.

11. Nicolaou, K. C.; Montagnon, T.; Baran, P. S. Angew.Chem. Int. Ed. 2002, 41, 993.

12. Nicolaou, K. C.; Gray, D. L. F.; Montagnon, T.; Harrison, S. T. Angew. Chem. Int. Ed. 2002, 41, 996.

13. Derek, M.; Andey, A. R.; Van De Water, R. W.; Pettus, T. R. R. Org. Lett. 2002, 4, 285.

14. Nicolaou, K. C.; Mathison, C. J. N.; Montagnon, T. Angew. Chem. Int. Ed. 2003, 42, 4077.

15. Brock, E. D.; Lewis, D. M.; Yousaf, T. I.; Harper, H. H. WO 9951688, 1999.

16. Gazit, A.; App, H.; McMahon, G.; Chen, J.; Levitzki, A.; Bohmer, F. D. J. Med. Chem. 1996, 39, 2170.

17. Sehlstedt, U.; Aich, P.; Bergman, J.; Vallberg, H.; Norden, B.; Graslund, A. J. Mol. Biol. 1998, 278, 31.

18. Dailey, S.; Feast, J. W.; Peace, R. J.; Sage, I. C.; Till, S.; Wood, E. L. J. Mater. Chem. 2001, $11,2238$.

19. O’Brien, D.; Weaver, M. S.; Lidzey, D. G.; Bradley, D. D. C. Appl. Phys. Lett. 1996, 69, 881.

20. Yamamoto, T.; Sugiyama, K.; Kushida, T.; Inoue, T.; Kanbara, T. J. Am. Chem. Soc. 1996, $118,3930$.

21. Yamamoto, T.; Zhou, Z.-H.; Kanbara, T.; Shimura, M.; Kizu, K.; Maruyama, T.; Nakamura, Y.; Fukuda, T.; Lee, B.-L.; Ooba, N.; Tomaru, S.; Kurihara, T.; Kanno, T.; Kubota, K.; Sasaki, S. J. Am. Chem. Soc. 1996, 118, 10389.

22. Nurulla, I.; Yamaguchi, I.; Yamamoto, T. Polym. Bull. 2000, 44, 231. 
23. Yamamoto, T.; Lee, B.-L.; Kokubo, H.; Kishida, H.; Hirota, K.; Wakabayashi, T.; Okamoto, H. Macromol. Rapid Commun. 2003, 24, 440.

24. Dell, A.; William, D. H.; Morris, H. R.; Smith, G. A.; Feeney, J.; Roberts, G. C. K. J. Am. Chem. Soc. 1975, 97, 2497.

25. Bailly, C.; Echepare, S.; Gago, F.; Waring, M. J. Anti-Cancer Drug Des. 1999, 15, 291.

26. Eicher, T.; Hauptmann, S. The Chemistry of Heterocycles; Thieme: New York, 1995; 417, 434.

27. Barluenga, J.; Aznar, F.; Liz, R. Cabal, M.-P. Synthesis 1985, 313.

28. Petukhov, P. A.; Tkachev, A. V. Tetrahedron 1997, 53, 9761.

29. Brown, D. J. Quinoxalines: Supplement II. In The Chemistry of Heterocyclic Compounds; Taylor, E. C., Wipf, P., Eds.; John Wiley\& Sons: New Jersey, 2004.

30. Mao, L. Sakurai, H. Hirao, T. Synthesis 2004, 2535.

31. Zhao, Z.; Wisnoski, D. D.; Wolkenberg, S. E.; Leister, W. H.; Wang, Y.; Lindsley, C. W. Tetrahedron Lett. 2004, 45 , 4873.

32. Bhosale, R. S.; Sarda, S. R.; Ardhapure, S. S.; Jadhav, W. N.; Bhusare, S. R.; Pawar, R. P. Tetrahedron Lett. 2005, 46, 7183.

33. More, S. V.; Sastry, M. N. V.; Wang, C. -C.; Yao C.-F. Tetrahedron Lett. 2005, 46, 6345.

34. (a)Heravi, M. M.; Keivanlo, A.; Rahimzadeh, M.; Bakavoli, M.; Ghassemzadeh, M. Tetrahedron Lett. 2004, 45, 5747. (b) Heravi, M. M.; Keivanlo, A.; Rahimzadeh, M.; Bakavoli, M.; Ghassemzadeh, M. Tetrahedron Lett. 2005, 46, 1607. (c) Heravi, M. M.; Derikvand, F. J. Mol. Catal. A. 2005, 242, 173. (d) Heravi, M. M.; Bakhtiari, Kh.; Bamoharram, F. F. Catal. Commun. 2006, 7, 373. (e) Heravi, M. M.; Nami, N.; Oskooie, H. A.; Hekmatshoar, R. Phosphorus Sulfur and Silicon 2006, 181, 87. (f) Heravi, M. M.; Tajbakhsh, M.; Ahmadi, A. N.; Mohajerani, B. Monatsh. Chem. 2006, 137, 175. (g) Heravi, M. M.; Derikvand, F.; Oskooie, H. A.; Hekmatshoar, R. Synth. Commun. 2006, 36, 77.

35. Heravi, M. M.; Taheri, Sh.; Bakhtiari, Kh.; Oskooie, H. A. Catal. Commun. 2006, in press. 